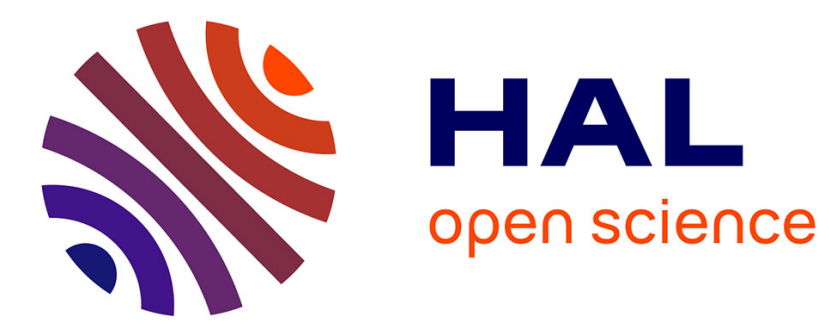

\title{
Modelling and simulation of powder-snow avalanches
}

\author{
Jocelyn Etienne, Marie Rastello, Emil J. Hopfinger
}

\section{To cite this version:}

Jocelyn Etienne, Marie Rastello, Emil J. Hopfinger. Modelling and simulation of powder-snow avalanches. Comptes Rendus Mécanique, 2006, 334 (8-9), pp.545 - 554. 10.1016/j.crme.2006.07.010 . hal-00623070

\section{HAL Id: hal-00623070 \\ https://hal.science/hal-00623070}

Submitted on 16 Sep 2011

HAL is a multi-disciplinary open access archive for the deposit and dissemination of scientific research documents, whether they are published or not. The documents may come from teaching and research institutions in France or abroad, or from public or private research centers.
L'archive ouverte pluridisciplinaire HAL, est destinée au dépôt et à la diffusion de documents scientifiques de niveau recherche, publiés ou non, émanant des établissements d'enseignement et de recherche français ou étrangers, des laboratoires publics ou privés. 


\title{
Modelling and simulation of powder-snow avalanches
}

\author{
Jocelyn Étienne ${ }^{1,+}$, Marie Rastello ${ }^{2}$ and Emil J. Hopfinger ${ }^{3, \#}$ \\ ${ }^{1}$ LMC IMAG, BP 53, 38041 Grenoble Cedex \\ ${ }^{+}$Present address: DAMTP, University of Cambridge, Wilberforce Road, Cambridge CB3 \\ OWA, GB \\ ${ }^{2}$ LMFA-ECL, 36, avenue Guy de Collongue, 69134 Ecully Cedex \\ ${ }^{3}$ LEGI/CNRS, B.P. 53, 38041 Grenoble Cedex 9 \\ ${ }^{*}$ Corresponding author. Telephone: +33 4768250 43, Fax: +33 476825271 \\ E-mail addresses: J.Etienne@damtp.cam.ac.uk (J. Étienne), Marie.Rastello@ec-lyon.fr (M. \\ Rastello), Emil.Hopfinger@hmg.inpg.fr (E.J. Hopfinger)
}

\begin{abstract}
Finite volume release gravity currents of large density contrast on steep slopes, representing powder-snow avalanches, are simulated numerically using a dynamic mesh adaptation technique. This technique allows to treat large Reynolds numbers and large density contrast flows but it is (presently) restricted to two dimensions. Comparison of numerical results with experiments in the Boussinesq limit shows that $2 \mathrm{D}$ simulations capture the essential dynamics. The physics of powder-snow avalanches is analysed on hand of the similarity model developed by Rastello and Hopfinger [1] and briefly reproduced here. The numerical simulations provide the closure parameters required in this model and give access to the flow structure. The non-Boussinesq effect is to decrease substantially the spatial growth in height and to increase the aspect ratio, hence the overall flow structure.
\end{abstract}

\section{Introduction}

Powder-snow avalanches are dense clouds moving down steep slopes. The extra weight of the cloud is due to the suspended snow particles in air of volume concentration 0.4 to $4 \%$. This results in a bulk density of about 2 to 30 times the air density. During the motion, air is entrained which leads to a dilution and growth of the cloud and, depending on the flow conditions, snow can also be picked-up from the underlying snow cover which may cause a continuous acceleration of the avalanche. In order to reach velocities of the order of $100 \mathrm{~m} / \mathrm{s}$, a value often quoted for powder-snow avalanches [2, 3], a continuous down-slope acceleration is required. This large front velocity and, consequently, the large turbulent velocity, assures that the snow particles of diameter $d_{p} \approx 0.5$ to $1 \mathrm{~mm}$ and density $\rho_{p} \approx 400$ to $900 \mathrm{~kg} / \mathrm{m}^{3}$ remain suspended and that an avalanche is capable of picking up further snow from the snow cover as long as the volume concentration is less than the saturation concentration. Furthermore, the Stokes number $S t=\tau_{p} / \tau_{f}$, expressing the characteristic particle time scale $\tau_{p}$ to flow time scale $\tau_{f} \approx H / U_{f}$, where $U_{f}$ is the avalanche front velocity and $H$ its height, is less than 0.1 , noting that because of the relatively large particle Reynolds numbers, $\tau_{p}$ is evaluated for a particle drag larger than the Stokes drag. For the case of a particle stratified shear layer Meiburg et al [4] showed that when the Stokes number is about 0.1 the particle loading has an effect similar to that of a single phase stratified fluid. Therefore, it can be assumed that the suspension cloud behaves like a single phase gravity flow of large density difference.

Beghin et al [5] conducted laboratory experiments with finite volume release gravity currents, forming dense clouds on slopes, using small density ratio (Boussinesq) saline solutions. Rastello and Hopfinger [1] (from here on referred to as RH) did similar experiments with saline solutions and in addition with finite volume release turbidity currents moving over 
a sediment layer on slopes. The turbidity clouds showed a similar behaviour to the single phase saline clouds. A similarity model has been developed by RH which contains as closure parameters an air (ambient fluid) entrainment coefficient $E_{c}$ and a sediment entrainment coefficient $E_{s}$. An additional parameter is the aspect ratio of cloud length to height $k$. These coefficients were obtained from the experiments in the Boussinesq limit and used in the model to predict avalanche velocity variation, noting that the avalanche velocity depends on $E_{c}$ and $E_{s}$ but not on $k$.

Further insight can be gained from direct numerical simulations especially in the case of large density ratios. It has been shown by Étienne et al [6] that large Reynolds number simulations of large density ratio gravity flows are possible by using a dynamic grid adaptation technique. The drawback is that these techniques are presently limited to twodimensions. Although the turbulence structure is three-dimensional, it can be assumed that the dynamics of the cloud movement is a two-dimensional process, controlled by the large scale structures of principal vorticity component normal to flow direction. Two-dimensional simulations of gravity currents [7] and of lock-exchange flows [8,6] for instance are in good agreement with experiments although the turbulence structure is three-dimensional.

In Section 2 the similarity model is presented and compared with measurements of the front velocity reported for the Sion Valley avalanche by Dufour et al [9]. In Section 3 the 2D numerical simulations are first applied to Boussinesq clouds for conditions corresponding to the experiments of RH. Then, numerical results are presented for the same conditions but with the density ratio increased to 20 . These results show clearly the non-Boussinesq effect on the flow structure; the acceleration length is substantially larger and the growth rate is reduced.

\section{Similarity model}

To a good approximation a powder-snow avalanche can be considered as a single phase dense cloud moving down steep slopes. Arguments supporting this assumption are developed above in Section 1. During the motion the size increases and, for a given slope angle, the shape remains similar, that is the aspect ratio of height to length remains constant ${ }^{1}$. Fig. 1 shows a schematic representation of the cloud shape with the definition of the relevant variables.

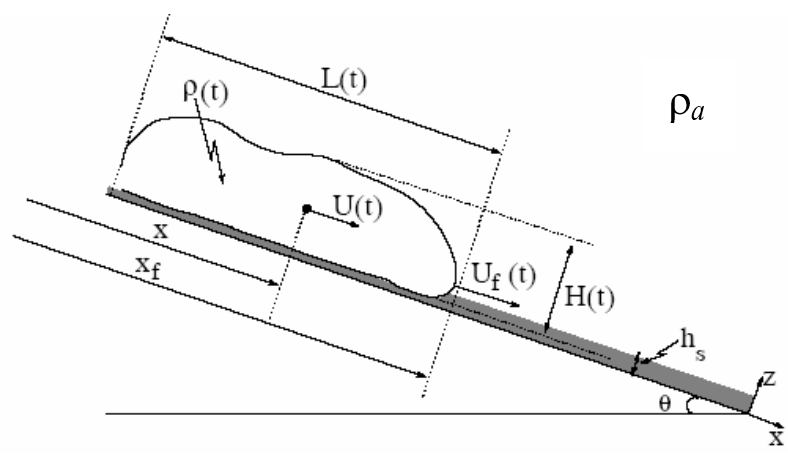

Fig. 1. Definition sketch

\footnotetext{
${ }^{1}$ This is a first approximation because, as the present numerical simulations indicate, the aspect ratio depends on density ratio which decreases with distance.
} 
The momentum, mass and volume per unit width conservation equations are respectively:

$$
\begin{aligned}
& \frac{d\left(\rho_{a} k_{v}+\rho\right) A U}{d t}=\Delta \rho A g \sin \theta-C_{f} \rho_{b} U^{2} L \\
& \frac{d \Delta \rho A}{d t}=\beta \Delta \rho_{s} h_{s} U=E_{s} \Delta \rho_{s} L U \\
& \frac{d A}{d t}=E_{c} U \sqrt{A}=E_{c} \frac{\sqrt{S_{1}}}{S_{2}} U P,
\end{aligned}
$$

where $\rho=\rho_{p} C+\rho_{a}(1-C), \Delta \rho=\rho-\rho_{a}, A=S_{1} k H^{2}, P=S_{2} \sqrt{H L}, k(\theta)=L / H, C_{f}$ is the bottom friction coefficient, $h_{s}$ the snow cover depth, $\beta$ is the fraction of the snow cover entrained along the avalanche path, $S_{1}$ and $S_{2}$ are shape factors, $k_{v}$ is the added mass coefficient and $E_{c}$ and $E_{s}$ are air (ambient fluid) and snow (sediment) entrainment coefficients respectively. The subscript $a$ stands for air or ambient fluid, $p$ for particles, $s$ for snow cover (sediment) and $b$ for bed. After transforming time into space by setting $U=d x / d t$ and neglecting bottom friction (term in $C_{f}$ ), equation (1) can be integrated (see [1]) in the form:

$$
U=\frac{\sqrt{2 K M x+(K B+G M) x^{2}+\frac{2}{3}(K N+G B) x^{3}+\frac{1}{2} G N x^{4}}}{M+B x+N x^{2}}
$$

where

$$
\begin{aligned}
& K=\left(\Delta \rho_{0} A_{0}\right) g \sin \theta \\
& M=\left(k_{v}+1\right) \rho_{a} A_{0}+\Delta \rho_{0} A_{0} \\
& N=\left(1+k_{v}\right) \frac{\rho_{a}}{4} E_{c}^{2} \\
& B=\beta \Delta \rho_{s} h_{s+}\left(1+k_{v}\right) \rho_{a} E_{c} A_{0}^{1 / 2} \\
& G=\beta \Delta \rho_{s} h_{s} g \sin \theta
\end{aligned}
$$

In the integration of equation (1) it was assumed that at $x=0, U=0$ but $A=A_{0}$ and $L_{0} / H_{0}=k$.

Equation (2) gives (keeping $S_{1}$ constant):

$$
H=H_{0}+\frac{E_{c}}{2 \sqrt{k S_{1}}} x
$$

and from equation (3) the buoyancy variation with distance is obtained in the form:

$$
\Delta \rho A=\Delta \rho_{0} A_{0}+\beta \Delta \rho_{s} h_{s} x
$$

Typical values for a powder-snow avalanche, the Sion Valley avalanche for instance [9] are: $A_{0}=200 \mathrm{~m}^{2}, \Delta \rho_{0}=150 \mathrm{~kg} / \mathrm{m}^{3}$, average slope angle $\theta=25^{\circ}, \Delta \rho_{\mathrm{s}}=150 \mathrm{~kg} / \mathrm{m}^{3}, h_{s}=1 \mathrm{~m}$. The shape factors are closely approximated by an elliptic shape, namely $S_{l} \cong 0.8$ and $S_{2} \cong 2.4$, while $k_{v}$ is taken constant, equal to 0.5 . It has been shown by RH that the velocity predicted 
by this similarity model is in good agreement with measured avalanche velocities when taking for the closure parameters $E_{c}$ and $\beta$, respectively the values $E_{c} \approx 0.25$ (for $\theta=25^{\circ}$ ) and $\beta=1$, i.e. the whole snow cover is incorporated along the avalanche path. Bottom friction is practically unimportant on steep slopes. This is demonstrated by integrating equation (1) numerically with and without bottom friction. It is, however, the principal retarding force when the avalanche moves onto nearly horizontal ground. Figure 2 shows a comparison of the model predictions (neglecting bottom friction) with measurements. In this figure the front velocity $U_{f}$ rather than the mass centre velocity is plotted because this velocity is directly accessible to measurements. The two are related by the spatial growth in cloud length. For the prediction of this spatial growth of an avalanche, it is necessary to know, in addition to the air entrainment coefficient $E_{c}$, the aspect ratio $k$. Observations suggest that $\mathrm{k} \approx 6$ for the Sion Valley avalanche.

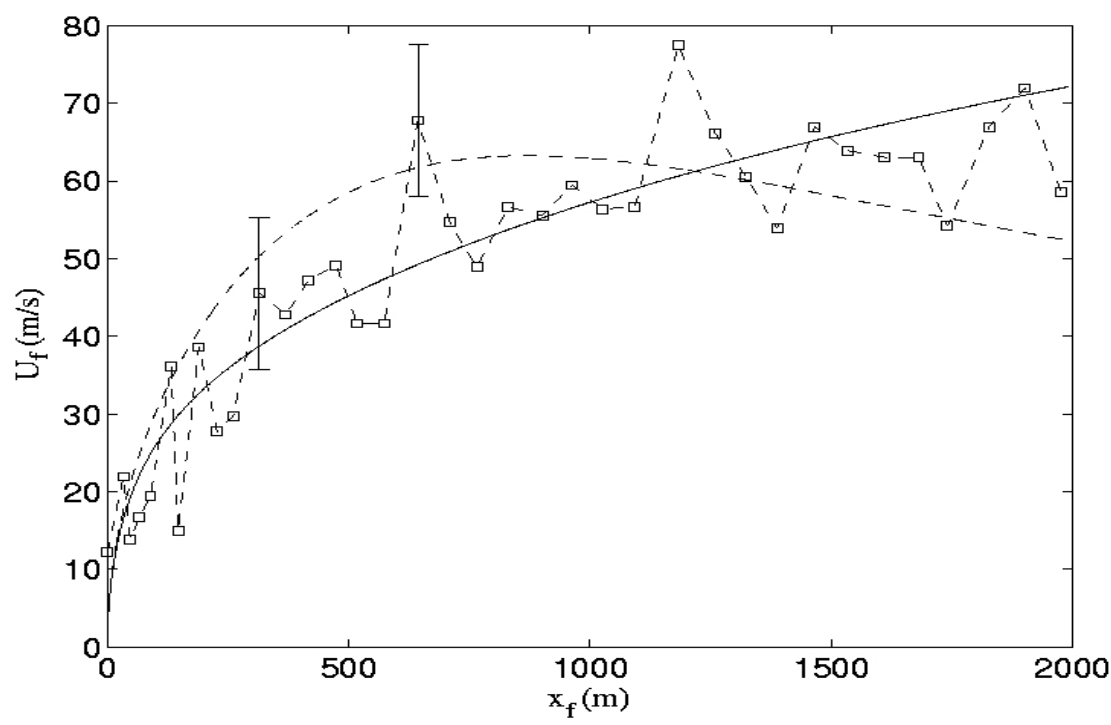

Fig. 2. Avalanche front velocity $U_{f}=U\left(1+E_{c} k^{1 / 2} / 4 S_{l}^{1 / 2}\right)$ as a function of front position. - $\square$-, measured avalanche front velocity [9]; - , model prediction for $\theta=25^{\circ}, E_{c}=0.25$, $\beta=1$ and $C_{f}=0 ;---$, model prediction for $\beta=0$ (no snow entrainment).

Field observations of powder-snow avalanches are difficult and rare and measurements of the variation of the front velocity with distance became available only recently [9]. Measurements of velocity and density distributions inside an avalanche are, however, nearly impossible, although some attempts were made using radar. Turbulence closure models (turbulent energy-dissipation models in particular), are used for practical applications and give useful information for quasi-steady flows [10] but give no information about the time dependency of local quantities (density and velocity variations). For this reason, and in order to understand the avalanche dynamics in more detail, we turn to laboratory experiments and numerical simulations. Laboratory experiments are, unfortunately, limited to Boussinesq flows $[11,5,1]$. Only lock-exchange flow experiments have been performed for density ratios of about 20 using gases [12]. It is hardly feasible to use gases for gravity current or dense cloud experiments on slopes because of the large gas volumes needed. Direct numerical simulations or large eddy simulations are, therefore, necessary tools of research in this area.

By using the automatic mesh adaptation technique, developed for gravitational flows by Étienne et al [6], it is possible to reach large Reynolds numbers (order $10^{5}$ ) and large density differences in direct numerical simulation. Since direct numerical simulations of dense clouds on steep slopes seem not to have been conducted previously it is necessary to validate 
numerical results by experiments at least in the Boussinesq limit. For this reason the conditions of the numerical simulations presented in the following Section 3.3 are the same as in the experiments of $\mathrm{RH}$. The simulations are then extended to a density ratio of 20 to quantify the non-Boussinesq effect.

\section{Numerical simulations}

\subsection{Governing equations}

We consider the isothermal flow of a single phase fluid with large density variations, and equal, constant dynamic viscosity $\eta$ under the conditions described above. For a perfect mixture of two incompressible fluids, of density $\rho_{d}$ (the heavier one) and of density $\rho_{a}$ (the lighter one), the local density is $\rho=\rho_{d} \Phi+\rho_{a}(1-\Phi)$, where $\Phi$ is the local volume fraction of the fluid of density $\rho_{d}$. The density contrast is defined as $\alpha=\left(\rho_{d}-\rho_{a}\right) / \rho_{a}$, and the density can be rewritten as $\rho=\rho_{a}(1+\alpha \Phi)$. Lengths are non-dimensionalized according to the scale $L_{r}=\sqrt{A_{0}}$ of initial release, $\tilde{x}=x / L_{r}$ and velocities by the terminal velocity $U_{r}=\sqrt{\alpha g L_{r}}$ of a fluid element of density $\rho_{d}$ in the ambient fluid of density $\rho_{a}, \tilde{\mathbf{u}}=\mathbf{u} / U_{r}$. Times are nondimensionalized as $\tilde{t}=t U_{r} / L_{r}$. Momentum and mass conservation of the cloud and ambient air are given by the Navier-Stokes equations,

$(1+\alpha \Phi) \frac{D \widetilde{\mathbf{u}}}{D \widetilde{t}}=-\nabla p+\frac{1}{\operatorname{Re}} \operatorname{div}\left(2 \mathrm{D} \widetilde{\mathbf{u}}-\frac{2}{3} \operatorname{div} \widetilde{\mathbf{u}} I\right)-\frac{1+\alpha \Phi}{\alpha} \mathbf{k}$,

$\alpha \frac{D \Phi}{D \widetilde{t}}+(1+\alpha \Phi) \operatorname{div} \widetilde{\mathbf{u}}=0$

where the Reynolds number ${ }^{2}$ is defined as $\mathrm{Re}=\rho_{a} U_{r} L_{r} / \eta$, and $\mathrm{D} \widetilde{\mathbf{u}}=\left(\nabla \widetilde{\mathbf{u}}+\nabla \widetilde{\mathbf{u}}^{T}\right) / 2$. Since the fluids are miscible, we cannot assume $D \rho / D t=0$, but rather that there are mass diffusion fluxes in the flow governed by Fick's law. This yields [6]:

$$
\frac{D \Phi}{D \widetilde{t}}+\Phi \operatorname{div} \widetilde{\mathbf{u}}=\frac{1}{\operatorname{ReSc}} \nabla^{2} \Phi
$$

where the Schmidt number is defined as $\mathrm{Sc}=\eta / \rho_{a} \mathcal{D}$, with $\mathscr{D}$ a reference diffusivity. Initial conditions consist of given distribution of $\rho$ initially, the velocity being set to zero everywhere. No inflow or outflow across the boundaries of the flow domain has been considered, thus boundary conditions are always $\nabla \Phi \cdot \mathbf{n}=0$, with either no-slip boundary conditions $\left(\tilde{\mathbf{u}}_{\mid \partial \Omega}=0\right)$ or zero wall friction $(\tilde{\mathbf{u}} \cdot \mathbf{n}=0$ and $\sigma \cdot \mathbf{n}-[(\sigma \cdot \mathbf{n}) \cdot \mathbf{n}] \mathbf{n}=\mathbf{0}$, where $\mathbf{n}$ is the wall

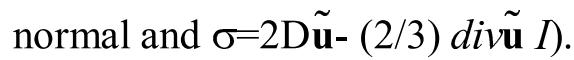

System $(7,8,9)$ was proposed by Étienne et al. [6] for the simulation of gas mixtures of high density contrast. An algorithm was devised for its resolution in the framework of finite elements and used for simulations of lock-exchange flows in two dimensions. These flows are a good test for high density contrast flow simulation, because there have been experimental and theoretical studies, and measurements for density contrasts up to $\alpha \approx 20$ are available [12]. Numerical results proved to be in good agreement with these measurements.

\footnotetext{
${ }^{2}$ Note that the choice of the reference velocity $U_{r}=\sqrt{\alpha g L_{r}}$ implies that the Froude number is simply $\sqrt{\alpha}$ in the equations.
} 


\subsection{Numerical technique}

Due to high Reynolds numbers and the flow structure, the flow of the buoyant clouds considered in this paper present features of very different spatial scales (time dependent strong local velocity and density gradients), which make dynamic mesh adaptation a necessity. Though this technique is still poorly developed in 3D [13], and the very high computational cost of three-dimensional DNS limits other approaches to Reynolds number lower than $10^{3}$ [7]. The numerical simulations presented here are thus restricted to the two dimensions $x$ and $z$, and make use of the dynamic mesh adaptation features of the mesh generator BAMG [14].

The remaining difficulty is to simulate efficiently both the transport and viscous terms on a general mesh. This is done by the Lagrange-Galerkin method [15], which consists in using the method of characteristics to discretise directly the material derivative along with finite elements for the discretisation in space. Étienne and Saramito [16] have designed an algorithm for Eqs. $(7,8,9)$ and shown optimal error estimates, and have implemented it in the open source free software RHEOLEF [17]. The finite element spaces are continuous, piecewise quadratic for the velocity and volume fraction, and continuous, piecewise linear for the pressure. The mesh refinement is an iterative process at each time step: a first estimate of the solution is calculated on a coarse mesh, and then the mesh is repeatedly refined and the solution recalculated until some mesh invariance is achieved. This is usually done in four remeshing iterations, with the final mesh having refinement ratios of order $10^{3}$ between the coarsest triangle size and the finest one, with an approximate total of $5 \times 10^{5}$ degrees of freedom.

\subsection{Results for Boussinesq clouds}

The simulations are first performed for a Boussinesq cloud which allows comparison with experiments. A volume of fluid of density $\rho_{d}$ and length $l_{0}=20 \mathrm{~cm}$ and $h_{0}=6.5 \mathrm{~cm}, A_{0}=$ $h_{0} l_{0}=130 \mathrm{~cm}^{2}$ and reference length $L_{r}=\sqrt{A_{0}}=11.4 \mathrm{~cm}$, is suddenly released in a stagnant fluid of density $\rho_{a}, \alpha=\left(\rho_{d}-\rho_{a}\right) / \rho_{a}=0.02$, and of depth $6 L_{r}$ and length $20 L_{r}$. The slope angle is $\theta=32^{\circ}$.

Figure 3 shows a qualitative comparison of the density contours. What is of interest here is the similar shape indicating a length to height ratio $k$ of about 3 . It should be noted, however, that there are large fluctuations in $k$ (about $+/-10 \%$ ) due to the time variations of the large eddy structure.

In Fig. 4 the simulated front velocity is compared with the experimental values of RH. There is good agreement when $\operatorname{Re}=U_{r} L_{r} \rho_{a} / \eta=10^{5}$, where $U_{r}=\sqrt{\alpha g L_{r}}$. In the simulation free slip conditions on the bottom were used (this corresponds to $C_{f}=0$ in the model). The calculated and experimentally determined spatial growth rates are compared in Fig. 5. There is reasonably good agreement in a range around the velocity maximum $\left(2<x_{f} / L_{r}\right.$ $<9$ ). At large distances the 2D simulations give a larger growth rate. This is to be expected since in 2D the large eddies are more persistent than in 3D. Note that $x_{f}$ is measured from the gate of release of the dense fluid. The virtual origin was taken as $x_{0}=-3 L_{r}$ in all the plots presented here. 


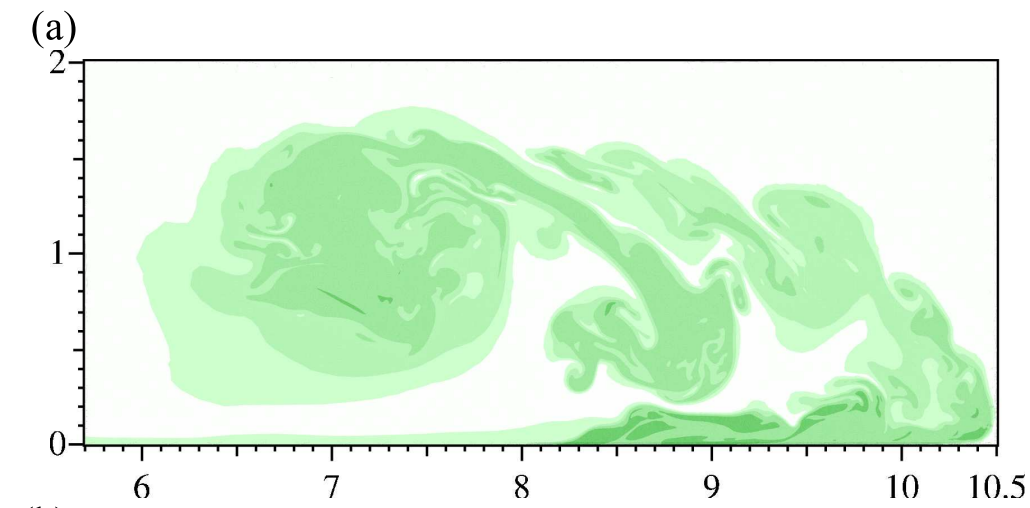

(b)

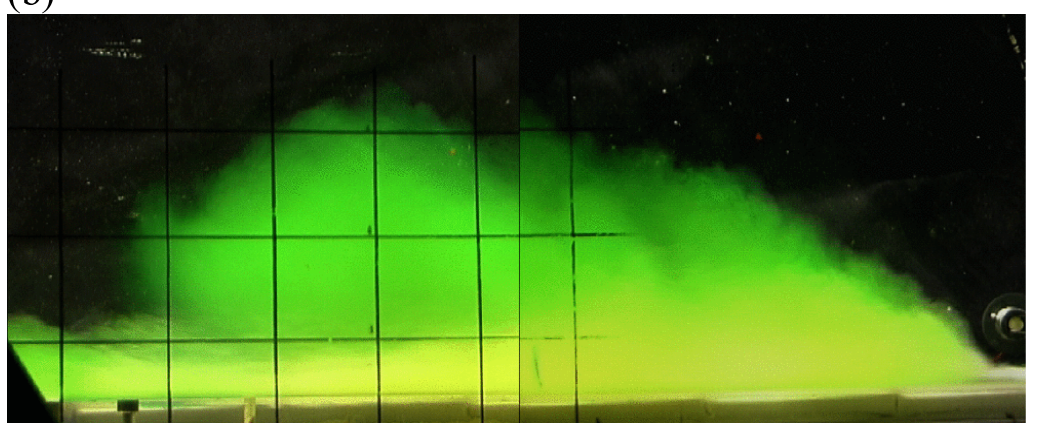

Fig. 3. Aspect of the cloud, (a), numerical simulation with $\alpha=0.02$ and $\mathrm{Re}=10^{5}$, (b), Experiments [1]. In the experiments the grid spacing is $5 \mathrm{~cm}$ and in (a) the coordinates are non-dimensionalized by $L_{r}=11.4 \mathrm{~cm}$.

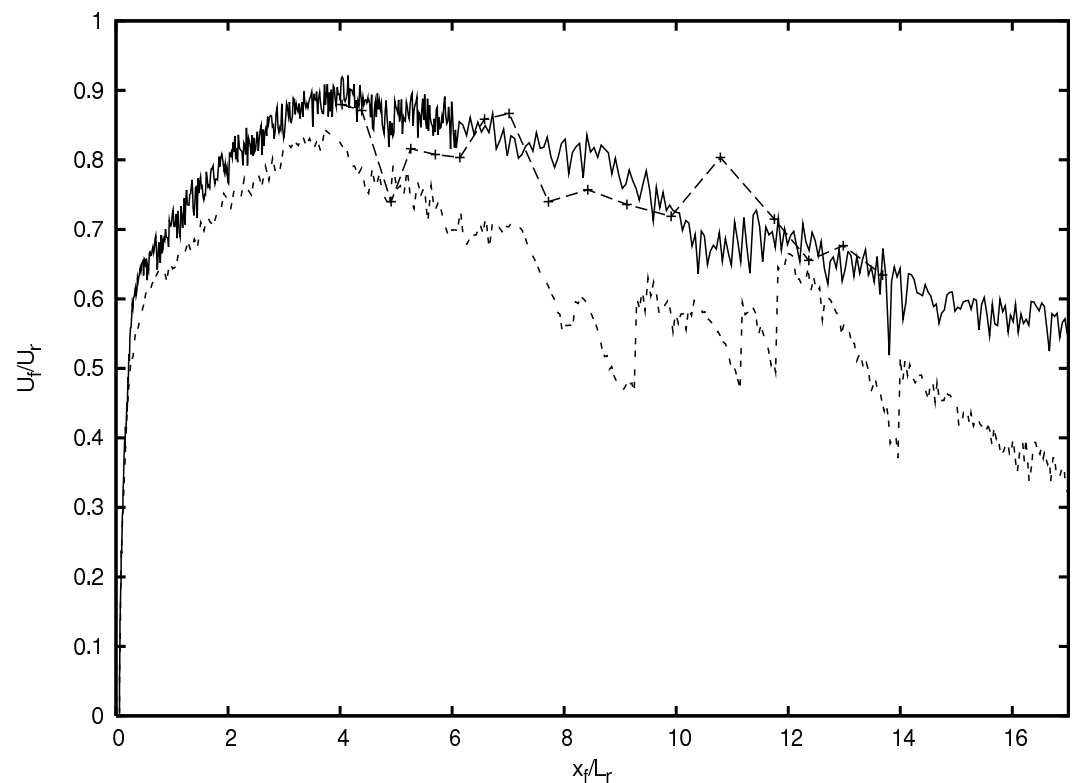

Fig. 4. Front velocity vs. front position, - Numerical simulation with $\alpha=0.02$ and $R e=10^{5}$, -- --, Numerical simulation with $\alpha=0.02$ and $\mathrm{Re}=10^{4},-+-$, Experimental results of $\mathrm{RH}$, with $\mathrm{Re}_{\exp }=\rho_{a} L_{r} \sqrt{\alpha g L_{r}} / \mu_{a} \approx 3 \times 10^{4}$. 


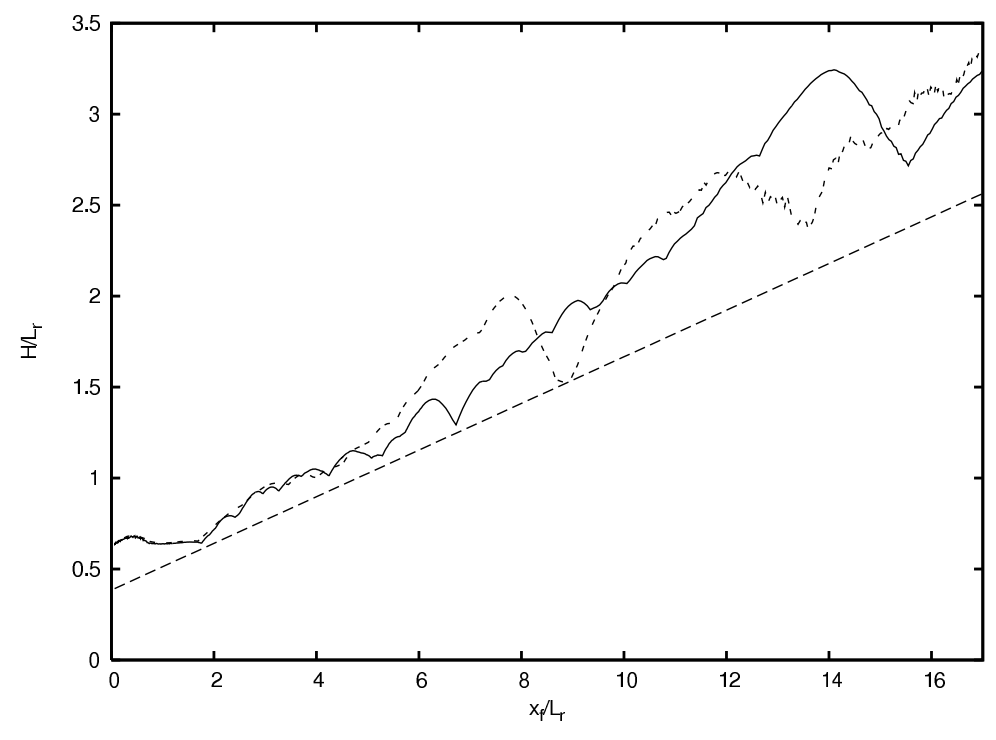

Fig. 5. Height of the cloud vs. front position, - Numerical simulation with $\alpha=0.02, \theta=32^{\circ}$ and $\mathrm{Re}=10^{5}$, -- --, Numerical simulation with $\alpha=0.02$ and $\mathrm{Re}=10^{4},--$, Experimental results of RH approximated by $\frac{d H}{d x_{f}}=3.6 \times 10^{-3} \theta+0.013$ ( $\mathrm{H}$ is determined with respect to the virtual origin at $\left.x_{f}=-3 L_{r}\right)$.

\subsection{Results for non-Boussinesq clouds}

The non-Boussinesq flow simulations were conducted for $\alpha=19$ and a Reynolds number $\operatorname{Re}=10^{5}$. The flow domain was increased to $50 L_{r}$, while the depth is reduced to $4 L_{r}$. The initial volume released and the slope angle were kept the same as in the Boussinesq case allowing a direct comparison. Figure 6 shows an image of the density contrast of the flow; the change in flow structure is clearly seen by comparing this image with Fig. 3.

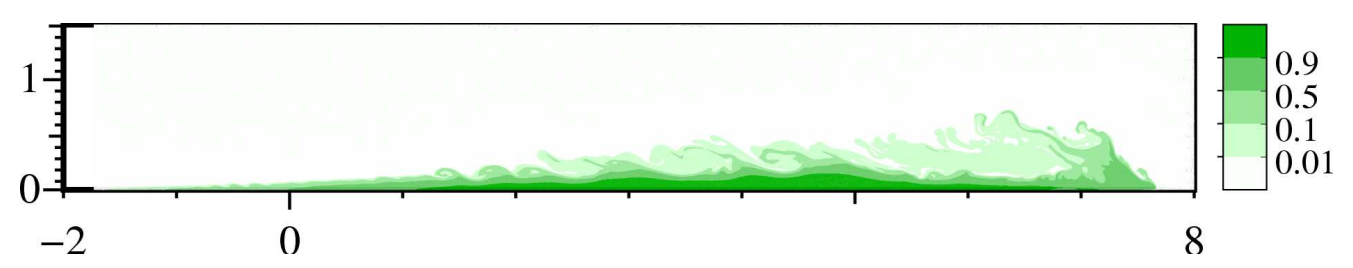

Fig. 6. Aspect of the cloud in a numerical simulation with $\alpha=19$ and $\mathrm{Re}=10^{5}$ : excess density $\Phi$ in colour code as a function of the space variables $x$ and $z$; coordinates are nondimensionalized by $L_{r}$.

In Fig. 7 the calculated mass centre velocity is plotted as a function of the position of the mass centre, non-dimensionalized by $L_{r}$. The calculated velocity for $\alpha=0.02$ as well as the solution of equations (1) to (3), taking $\beta=0, C_{f}=0$ and $E_{c}=0.26$ are presented for comparison. There is good agreement with the model but the calculated velocity decreases more rapidly than is predicted by the model. This may be due to a larger entrainment of ambient fluid in the 2D simulations. 


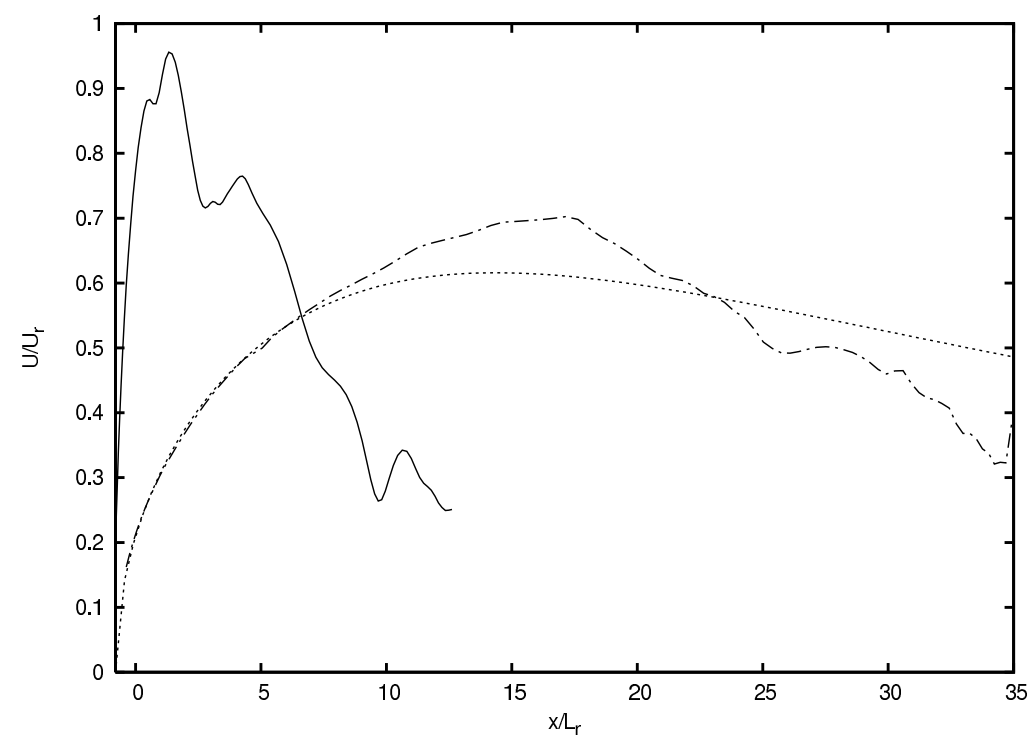

Fig. 7. Mass centre velocity of the cloud vs. non-dimensional mass centre position, ---, Numerical simulation for $\alpha=19, \theta=32^{\circ}$ and $\mathrm{Re}=10^{5}$; - , numerical simulation for $\alpha=0.02$ and $\mathrm{Re}=10^{5} ; \cdots$, integrated model equation (1) for no snow-entrainment, $\beta=0$ and $E_{c}=0.26$, $\mathrm{k}_{\mathrm{v}}=0.5, C_{f}=0$. The velocity is non-dimensionalized by $U_{r}=\sqrt{\alpha g L_{r}}$.

In Fig. 8 the non-dimensional height is plotted versus front position for $\alpha=0.02$ and $\alpha=19$. There is clearly a substantial decrease in the spatial growth rate of the cloud when the density ratio is large. The dashed straight line represents the experimentally determined growth rate approximated by $H=\left(3.6 \times 10^{-3} \theta+0.013\right)\left(x_{f}-x_{0}\right)$, and the dotted line corresponds to the extrapolation to a non-Boussinesq cloud proposed by $\mathrm{RH}$ given by $\left.\frac{d H}{d x}\right|_{N B}=\left.\frac{d H}{d x}\right|_{B} \frac{1+\sqrt{\rho_{a} / \rho_{b}}}{2}$, where subscripts NB and B stand for non-Boussinesq and Boussinesq. The model predicts reasonably well the decrease in growth rate when the density ratio is large (note that for the bed density $\rho_{b}$ the value of the initial density was used, $\rho_{b} / \rho_{a}=$ 20). RH assumed that the spatial growth in cloud length does not depend on density ratio. The numerical simulations confirm this assumption (Fig. 9). The aspect ratio of the avalanche (cloud), $k=L / H$ is, therefore, larger for large density difference clouds and is approximated by $k_{N B}=k_{B} \frac{2}{1+\sqrt{\rho_{a} / \rho_{b}}}$. Consequently the entrainment coefficient $E_{c}$ is reduced by $E_{c N B}=E_{c B} \sqrt{\left(1+\sqrt{\rho_{a} / \rho_{b}}\right) / 2}$. For a Boussinesq cloud on a slope of $32^{\circ}$ the value of $E_{c}$ is approximately 0.32 , which gives for the non-Boussinesq cloud with $\alpha=19$ a value of $E_{c} \approx$ 0.25. This justifies the value used for calculating the front velocity in Fig . 7. 


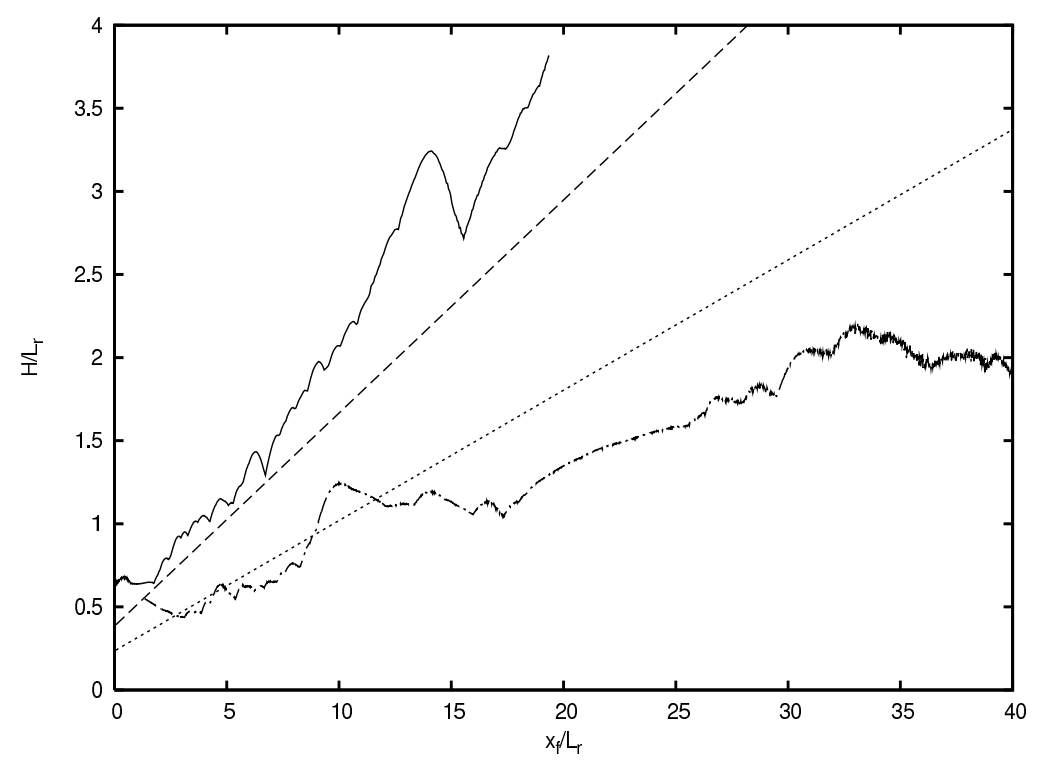

Fig. 8. Non-dimensional height of the cloud vs. front position. - , numerical simulation with $\alpha=0.02$ and $\operatorname{Re}=10^{5},-\cdot-$, Numerical simulation with $\alpha=19$ and $\operatorname{Re}=10^{5}$, - - Experimental results of RH for Boussinesq clouds, -. Extrapolation of experimental results to nonBoussinesq conditions.

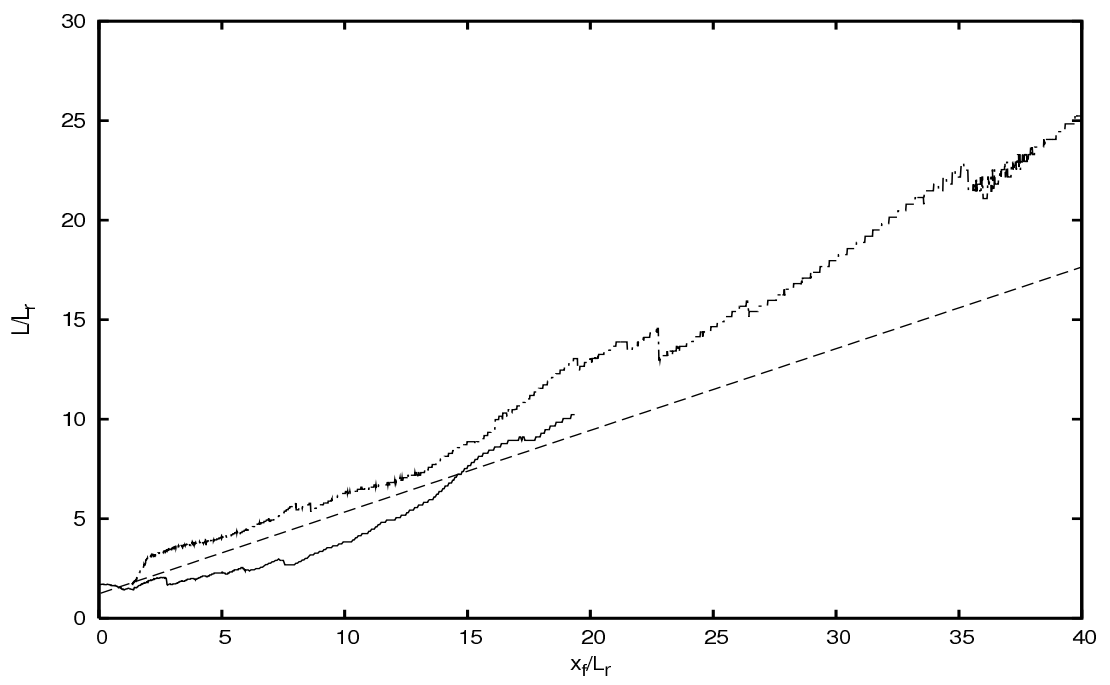

Fig. 9. Non-dimensional length of the cloud vs. front position. - , numerical results for $\alpha=$ $0.02 ;--, \alpha=19$. The dashed straight line corresponds to $L=k H$ with $k_{N B}=k_{B} \frac{2}{1+\sqrt{\rho_{a} / \rho_{b}}}$.

\section{Conclusions}

To our knowledge the simulations presented in this paper are the first numerical simulations of large density difference, finite volume release gravity currents on steep slopes forming dense clouds. The finite element scheme used with dynamic mesh adaptation allows to reach sufficiently large Reynolds numbers but simulations are limited to two dimensions. A comparison of numerical results with experiments in the Boussinesq limit shows that the essential physics is well captured by 2D such simulations. The simulations apply to powdersnow avalanches under the assumption that avalanches can be treated as a single phase, large density difference flow. This assumption is justified by the value of the Stokes number ( $\mathrm{St}<$ 
0.1 ) and is further supported by the similarity model presented in Section 2 which compares well with measured avalanche velocities. The closure parameters in this similarity model were determined from laboratory experiments in the Boussinesq limit and extrapolated to nonBoussinesq clouds by RH. To a good approximation the present numerical simulations support these theoretical extrapolations.

\section{References :}

[1] M. Rastello, E. J. Hopfinger, Sediment-entraining suspension clouds: a model of powdersnow avalanches, J. Fluid Mech. 509 (2004) 181-206.

[2] E. J. Hopfinger, Snow avalanche motion and related phenomena, Ann. Rev. Fluid Mech. 15 (1983) 47-76.

[3] K. Hutter, Avalanche dynamics, in: V. P. Singh (Ed.), Hydrology of disasters, Kluwer Academic Publishers, Dordrecht etc., 1996.

[4] E. Meiburg, E. Wallner, A. Pagella, A. Riaz, C. J. J. Haertel, F. Necker, Vorticity dynamics of dilute two-way-coupled particle-laden mixing layers, J. Fluid Mech. 421 (2000) $185-227$.

[5] P. Beghin, E. J. Hopfinger, R. E. Britter, Gravitational convection from instantaneous sources on inclined boundaries, J. Fluid Mech. 107 (1981), 407-422.

[6] J. Étienne, E. J. Hopfinger, P. Saramito, Numerical simulations of high density ratio lockexchange flows, Phys. Fluids 17 (2005) 036601.

[7] C. J. J. Haertel, E. Meiburg, F. Necker, Analysis and direct numerical simulation of the flow at a gravity-current head. Part 1. Flow topology and front speed for slip and no-slip boundaries, J. Fluid Mech. 418 (2000)189-212.

[8] V. K. Birman, J. E. Martin, E. Meiburg, The non-Boussinesq lock-exchange problem. Part 2: high resolution simulations, J. Fluid Mech. 537 (2005) 125-144.

[9] F. Dufour, U. Gruber, W. Ammann, Avalanches : études réalisées dans la Vallée de la Sionne en 1999, Les Alpes 2 (2001) 9-15.

[10] P. Sampl, T. Zwinger, 2004, Avalanche simulation with SAMOS, Annals Glaciol. 38 (2004) 393-398.

[11] R. E. Britter, P. F. Linden, The motion of the front of a gravity current travelling down an incline, J. Fluid Mech. 99 (1980) 531-543.

[12] H. P. Groebelbauer, T. K. Fanneløp, R. E. Britter, The propagation of intrusion fronts of high density ratios, J. Fluid Mech. 250 (1993) 669-687.

[13] P.-L. George, Sur la construction de maillages, Congres Français de Mecanique, Troyes, (2005).

[14] F. Hecht, BAMG : Bidimenisonal Anisotropic Mesh Generator. http://www-

rocq1.inria.fr/gamma/cdrom/www/bamg/eng1.htm, INRIA, Rocquencourt, France (1997).

[15] M. Bercovier, O. Pironneau, Characteristics and the finite elements method, in: Tadahiko Kawai (Ed.), Finite element flow analysis, proceedings of the 4th Int. Symp. on Finite Elements Methods in Flow Problems, Tokyo, North-Holland (1982) 67-73.

[16] J. Étienne, P. Saramito, A priori error estimates of the Lagrange-Galerkin method for Kazhikhov-Smagulov type systems, C.R. Acad. Sci. Paris, Ser. I, in press (2005).

[17] P. Saramito, N. Roquet, J. Étienne, RHEOLEF, a finite element environment. http://www-lmc.imag.fr/lmc-edp/Pierre.Saramito/rheolef, LMC, Grenoble, France, (2003). 